\title{
Efficiency Evaluation of Emergency Rescue System for Island Bridge and Tunnel cross Sea Cluster
}

\author{
Jian Wang, Wenjia Liu \\ School of Transportation Science and Engineering, Harbin Institute of Technology \\ Huanghe Road, No.73, Nangang District, Harbin, PR.China \\ wang_jian@hit.edu.cn; liuwenjia0826@outlook.com
}

\section{Extended Abstract}

The cross-sea cluster environment of Island Bridge and Tunnel is made up of a combination of long tunnels, artificial islands, and extra large bridges. Since the overall structure is a closed tunnel without any cross or sub-flow, in the event of emergencies, especially those involving dangerous goods Incidents, it will lead to paralysis across the board and affect the operation of the entire channel. Island Bridge cross-sea cluster rescue environment is more complex, including the rescue of the sea tunnel difficult, the cross-regional special bridge, the bridge unique environment and a series of special problems. Due to the complexity of the rescue environment, the influence of cross-regional coordination and other factors, the weight of each index in emergency rescue system evaluation is difficult to be determined, and the relational expression of each evaluation index can not be accurately calculated.

Based on the analysis of relevant research results both at home and abroad, this dissertation focuses on quantitative argumentation and complements qualitative analysis, and proposes a data envelopment analysis method. According to the basic elements of traffic emergency rescue, from the perspectives of eight elements (highway police force, number of medical staff, number of ambulances, number of fire engines, number of wrecked vehicles, arrival time of rescue supplies, success rate of personnel rescue and economic loss) A set of evaluation index system of emergency rescue system suitable for traffic environment of island bridge across the sea is constructed. In addition, in order to analyze the impact of unwanted output on the efficiency of decision-making unit, this paper proposes a method to deal with non-expected output, and carries out a case analysis of the efficiency evaluation model. In this paper, the island bridge tunnel cross-sea cluster environment to draw similar cases, according to China's major accident investigation report, each special accident as DUM evaluation unit, by calculating the Island Bridge cross-sea cluster environmental traffic emergency rescue system Comprehensive efficiency, we can see the comprehensive efficiency of emergency rescue system of emergency is 0.707 , which is not effective for non DEA. The pure technical efficiency is 0.97 , which is non DEA effective. The scale efficiency is 0.72 , which is non DEA effective. The decrease of scale returns shows that the efficiency of emergency rescue system has not reached the effective frontier, and the comprehensive efficiency is relatively poor. In order to be effective, it is necessary to adjust the allocation of emergency resources in emergency rescue system. The number of road police should be reduced by 21 and the number of medical staff should be reduced by 7 ; the number of ambulances and the number of fire engines should be reduced to 6 and 3 respectively while the number of vehicles with wrecked obstacles should be reduced to 2. As emergency supplies have been configured reserves, which can not be wasted, therefore they can be used as supplies reserve.

This article aims to provide valuable reference and experience for the road traffic management department and the emergency management department. Through the quantitative analysis of the efficiency of the emergency rescue system of traffic accident in cross-sea cluster environment of Island Bridge, it provides a theoretical basis for designing and setting a more reasonable traffic emergency rescue for the cross-sea cluster environment of Island Bridge.

Keywords: Transportation Engineering, Traffic Emergency, Data Envelopment Analysis. 